\title{
A RELATIVE BICOMMUTANT THEOREM: THE STABLE CASE OF PEDERSEN'S QUESTION
}

\author{
THIERRY GIORDANO AND PING W. NG
}

\begin{abstract}
In 1976, D. Voiculescu proved that every separable unital sub-C*algebra of the Calkin algebra is equal to its (relative) bicommutant. In his minicourse (see 17]), G. Pedersen asked in 1988 if Voiculescu's theorem can be extended to a simple corona algebra of a $\sigma$-unital $\mathrm{C}^{*}$-algebra. In this note, we answer Pedersen's question for a stable $\sigma$-unital $\mathrm{C}^{*}$-algebra.
\end{abstract}

\section{INTRODUCTION}

Let $\mathcal{H}$ be a separable infinite dimensional Hilbert space. Recall that the Calkin algebra, the quotient of $\mathbb{B}(\mathcal{H})$ by the closed two-sided ideal $\mathcal{K}=\mathcal{K}(\mathcal{H})$ of compact operators, is a (non separable) simple $\mathrm{C}^{*}$-algebra. In his ground-breaking publication on the noncommutative Weyl-von Neumann theorem, D. Voiculescu proved the following:

Theorem A. (21], Corollary 19) A separable unital sub-C*-algebra of the Calkin algebra is equal to its (relative) bicommutant.

For a $\mathrm{C}^{*}$-algebra $\mathcal{B}$, let $\mathcal{M}(\mathcal{B})$ denote its multiplier algebra and $\mathcal{M}(\mathcal{B}) / \mathcal{B}$ its corona $\mathrm{C}^{*}$-algebra. As the multiplier algebra of $\mathcal{K}$ is isomorphic to $\mathbb{B}(\mathcal{H})$, the corona $\mathrm{C}^{*}$-algebra of $\mathcal{K}$ is the Calkin algebra.

In his mini-course on the corona construction ([17]), G.K. Pedersen asks in Remark 10.11 if Voiculescu's theorem can be extended to the case of a $\sigma$-unital algebra $\mathcal{B}$, whose corona algebra is simple.

As the corona algebra of a $\sigma$-unital simple purely infinite $\mathrm{C}^{*}$-algebra is simple ([14, 15]), the following main result of this note gives a positive answer to the stable case of Pedersen's question:

Theorem B. Let $\mathcal{B}$ be a $\sigma$-unital stable simple and purely infinite $C^{*}$-algebra.

Then any separable unital sub-C $C^{*}$-algebra of $\mathcal{M}(\mathcal{B}) / \mathcal{B}$ is equal to its (relative) bicommutant.

This note is structured as follows. We quickly review in Section 2, ElliottKucerovsky theory of absorbing extensions and use it to show in Proposition 2.4 that a separable stable simple and purely infinite $\mathrm{C}^{*}$-algebra has a nice theory of extensions. This becomes one of the key tools of the proof of Theorem B.

Let $\mathcal{A}$ be a unital norm-closed subalgebra of a unital $\mathrm{C}^{*}$-algebra $\mathcal{D}$. Following 21, let $\operatorname{Lat}(\mathcal{A})$ denote the set of all self-adjoint projections $e$ of $\mathcal{D}$ such that $(1-e) a e=0$ for every $a \in \mathcal{A}$, and let $\mathcal{A l g}(\operatorname{Lat}(\mathcal{A}))$ be the subalgebra of all $y \in \mathcal{D}$ such that $(1-e) y e=0, \forall e \in \operatorname{Lat}(\mathcal{A})$. Notice that if $\mathcal{D}=\mathbb{B}(\mathcal{H})$, then $\operatorname{Lat}(\mathcal{A})$ is the lattice of closed invariant subspaces (projections) for $\mathcal{A}$, and $\mathcal{A l g}(\operatorname{Lat}(\mathcal{A}))=$

T. G. was partially supported by a grant from NSERC Canada. 
$\{T \in \mathbb{B}(\mathcal{H}) ; \operatorname{Lat}(\mathcal{A}) \subset \operatorname{Lat}(T)\}$. Then $\mathcal{A}$ is a reflexive subalgebra of $\mathbb{B}(\mathcal{H})$ if $\mathcal{A}=\mathcal{A l g}(\operatorname{Lat}(\mathcal{A}))$.

In Theorem 1.8 of [21, D. Voiculescu proves that if $\mathcal{A}$ is a separable unital normclosed subalgebra of the Calkin algebra, then $\mathcal{A}$ is equal to $\mathcal{A l g}(\operatorname{Lat}(\mathcal{A}))$. The main result of Section 3 is Theorem 3.4 where we generalizes Voiculescu's result and its proof by replacing $\mathcal{K}$ by any separable stable simple purely infinite $\mathrm{C}^{*}$-algebra $\mathcal{B}$. Corollary 3.5 is then the direct generalization of Theorem A

In Section 4, we generalize Theorem 3.4 by relaxing the separability condition on the $\mathrm{C}^{*}$-algebra $\mathcal{B}$ and prove Theorem $\mathrm{B}$ (as Corollary 4.4).

\section{Preliminaries in the Theory of ABsorbing EXtensions}

The theory of extensions and of absorbing extensions has a long history (see in particular [4, [5, [12, 16, 21]), culminating in the Elliott-Kucerovsky theory of absorbing extensions. In this section, we briefly sketch some preliminaries and recall Elliott-Kucerovsky's Theorem 6.

Using Lemma 2.3. we summarize in Proposition 2.4 several characterizations of a separable stable simple purely infinite $\mathrm{C}^{*}$-algebra using properties of its theory of extensions.

For background notions in the theory of extensions, we use monographs [2] and 20.

Definition 2.1. (See [5] Definitions 1 and 2.)

(1) Let $\mathcal{B}$ be a $C^{*}$-algebra and $\mathcal{E}$ a $C^{*}$-algebra containing $\mathcal{B}$ as a closed two sided ideal. We say $\mathcal{E}$ is purely large with respect to $\mathcal{B}$ if for all $d \in \mathcal{E}-\mathcal{B}$, the $C^{*}$-algebra $\overline{d \mathcal{B} d^{*}}$ contains a stable sub- $C^{*}$-algebra which is full in $\mathcal{B}$.

(2) Let $\mathcal{A}, \mathcal{B}$ be $C^{*}$-algebras and let

$$
0 \rightarrow \mathcal{B} \rightarrow \mathcal{E} \rightarrow \mathcal{A} \rightarrow 0
$$

be an extension of $\mathcal{B}$ by $\mathcal{A}$. We say that the extension is purely large if the $C^{*}$-algebra of the extension, $\mathcal{E}$, is purely large with respect to the image of $\mathcal{B}$ in it, in the sense described in (1).

Note that a purely large extension $0 \rightarrow \mathcal{B} \stackrel{\gamma}{\rightarrow} \mathcal{E} \stackrel{\pi}{\rightarrow} \mathcal{A} \rightarrow 0$ is essential (that is, $\gamma(\mathcal{B})$ is an essential closed two-sided ideal of $\mathcal{E})$.

Recall that to any extension $0 \rightarrow \mathcal{B} \rightarrow \mathcal{E} \rightarrow \mathcal{A} \rightarrow 0$ is naturally associated a *-homomorphism from $\mathcal{A}=\mathcal{E} / \mathcal{B}$ to the corona algebra $\mathcal{M}(\mathcal{B}) / \mathcal{B}$ called the Busby invariant of the extension. It is well-known (see, for example, [2, 15.4) that up to strong isomorphism (in the sense of Blackadar), an extension is determined by its Busby invariant. Therefore, we will not distinguish between extensions and their Busby invariants.

For the rest of this section, let $\mathcal{A}$ be a unital $\mathrm{C}^{*}$-algebra. Recall $([2,15.5)$ that a unital, extension $\phi: \mathcal{A} \rightarrow \mathcal{M}(\mathcal{B}) / \mathcal{B}$ is a (strongly) unital trivial extension if it lifts to a unital *-homomorphism $\phi_{0}$ from $\mathcal{A}$ to $\mathcal{M}(\mathcal{B})$. To simplify notation and as in [5], we will omit the term strongly.

As in [5], Definition 5, recall that a ${ }^{*}$-homomorphism $\phi_{0}: \mathcal{A} \rightarrow \mathcal{M}(\mathcal{B})$ is weakly nuclear if for each contraction $b \in \mathcal{B}$, the contractive c.p. map $\mathcal{A} \rightarrow \mathcal{B}$ given by $a \mapsto b \phi_{0}(a) b^{*}$ is nuclear (i.e., it factors approximately (in norm) through finite dimensional $\mathrm{C}^{*}$-algebras with contractive c.p. maps). Then a trivial unital extension $\phi: \mathcal{A} \rightarrow \mathcal{M}(\mathcal{B}) / \mathcal{B}$ is weakly nuclear if it lifts to a weakly nuclear unital *-homomorphism $\phi_{0}: \mathcal{A} \rightarrow \mathcal{M}(\mathcal{B})$. 
If $\mathcal{B}$ is stable, let $S$ and $T$ be two isometries of $\mathcal{M}(\mathcal{B})$ such that $S S^{*}+T T^{*}=$ $1_{\mathcal{M}(\mathcal{B})}$. Then for two extensions $\phi, \psi: \mathcal{A} \rightarrow \mathcal{M}(\mathcal{B}) / \mathcal{B}$, let $\phi \oplus \psi$ be their BrownDouglas-Fillmore (BDF) sum given, for $a \in \mathcal{A}$, by

$$
\phi \oplus \psi(a)=s \phi(a) s^{*}+t \psi(a) t^{*}
$$

where $s=q(S)$ and $t=q(T)$ (and $q$ is the canonical surjection from $\mathcal{M}(\mathcal{B})$ to $\mathcal{M}(\mathcal{B}) / \mathcal{B})$. The BDF sum is well-defined up to BDF-equivalence and is independent of the choice of isometries $S$ and $T$ in $\mathcal{M}(\mathcal{B})$.

Let $\mathcal{A}$ and $\mathcal{B}$ be separable $\mathrm{C}^{*}$-algebras with $\mathcal{A}$ unital and $\mathcal{B}$ stable. A unital extension $\phi: \mathcal{A} \rightarrow \mathcal{M}(\mathcal{B}) / \mathcal{B}$ is absorbing in the nuclear sense if for any weakly nuclear (strongly) unital trivial extension $\psi: \mathcal{A} \rightarrow \mathcal{M}(\mathcal{B}) / \mathcal{B}$, there exists a unitary $U \in \mathcal{M}(\mathcal{B})$ such that, for all $a \in \mathcal{A}$,

$$
\phi(a)=u(\phi \oplus \psi(a)) u^{*}
$$

where $u=q(U)$.

Then the main result of Elliott-Kucerovsky's paper is the following:

Theorem 2.2. ([5], Theorem 6) Let $\mathcal{A}$ and $\mathcal{B}$ be separable $C^{*}$-algebras with $\mathcal{A}$ unital and $\mathcal{B}$ stable.

For a unital essential extension $\phi: \mathcal{A} \rightarrow \mathcal{M}(\mathcal{B}) / \mathcal{B}$ of $\mathcal{B}$ by $\mathcal{A}$, the following statements are equivalent:

(1) $\phi$ is purely large.

(2) $\phi$ is absorbing in the nuclear sense.

For the sake of completeness, we include a proof of the following lemma, even if it is certainly well-known to the experts.

Lemma 2.3. Let $\mathcal{B}$ be a separable stable simple $C^{*}$-algebra with the following property:

For every unital separable $C^{*}$-algebra $\mathcal{A}$, every unital essential extension of $\mathcal{B}$ by $\mathcal{A}$ is absorbing in the nuclear sense.

Then the corona algebra $\mathcal{M}(\mathcal{B}) / B$ of $\mathcal{B}$ is simple.

Proof. We prove the contrapositive. Suppose that $\mathcal{M}(\mathcal{B}) / \mathcal{B}$ is not simple. Let $a \in \mathcal{M}(\mathcal{B}) / \mathcal{B}$ be a nonzero self-adjoint element which is not full in $\mathcal{M}(\mathcal{B}) / \mathcal{B}$, i.e., the closed two sided ideal generated by $a$ is a proper ideal of $\mathcal{M}(\mathcal{B}) / \mathcal{B}$. Let $\phi: C^{*}\left(a, 1_{\mathcal{M}(\mathcal{B}) / \mathcal{B}}\right) \rightarrow \mathcal{M}(\mathcal{B}) / \mathcal{B}$ be the inclusion map. Then $\phi$ is a unital essential extension. However, $\phi$ is not absorbing in the nuclear sense, since nuclearly absorbing extensions always bring nonzero elements to full elements (and since $\phi(a)$ is not full).

Recall that a nonunital $\sigma$-unital simple $\mathrm{C}^{*}$-algebra $\mathcal{B}$ has continuous scale if $\mathcal{B}$ has an approximate identity $\left\{e_{n}\right\}_{n=1}^{\infty}$ such that i. $e_{n+1} e_{n}=e_{n}$ for all $n$ and ii. for every $b \in \mathcal{B}_{+}-\{0\}$, there exists $N \geq 1$ such that for all $m>n \geq N, e_{m}-e_{n} \preceq b$. (See 14.)

We summarize and recall in the next proposition several characterizations of the simplicity of the corona algebra. This also illustrates the correspondence between "nice" extension theory and "nice" corona algebra structure.

Proposition 2.4. Let $\mathcal{B}$ be a separable stable simple $C^{*}$-algebra.

Then the following statements are equivalent: 
(1) Either $\mathcal{B} \cong \mathcal{K}$ or $\mathcal{B}$ is simple purely infinite.

(2) Either $\mathcal{B} \cong \mathcal{K}$ or $\mathcal{B}$ has continuous scale.

(3) $\mathcal{M}(\mathcal{B}) / \mathcal{B}$ is simple.

(4) $\mathcal{M}(\mathcal{B}) / \mathcal{B}$ is simple purely infinite.

(5) For every unital separable $C^{*}$-algebra $\mathcal{A}$, every unital essential extension of $\mathcal{B}$ by $\mathcal{A}$ is purely large.

(6) For every unital separable $C^{*}$-algebra $\mathcal{A}$, every unital essential extension of $\mathcal{B}$ by $\mathcal{A}$ is absorbing in the nuclear sense.

Proof. The equivalence of (2), (3) and (4) can be found in 14 and 15] (see also 22]). [15]).

The equivalence of (1) and (3) can be found in [23] and [18] (see also [14] and

The equivalence of (5) and (6) is [5] Theorem 6 (see our Theorem 2.2).

That (1) implies (5) can be found in [5] Theorem 17 items (i) and (ii), as well as 21.

That (6) implies (3) is Lemma 2.3 .

We note that in Proposition 2.4 above, statements (2), (3) and (4) are equivalent even without the assumption of stability (e.g., see [15] Corollary 3.3). We will require stability to relate simplicity of the corona algebra to simple pure infiniteness of the canonical ideal as well as nice extension theory.

\section{Generalization of Voiculescu's bicommutant theorem}

In this section, we first show three lemmas, which will be used in the proof of Theorem 3.4 and Corollary 3.5. These two results generalize Voiculescu's Theorem 1.8 and Corollary 1.9 of 21$]$.

Let us first recall some notation introduced in 21. If $\mathcal{D}$ is a unital $\mathrm{C}^{*}$-algebra and if $\mathcal{A}$ is a subalgebra of $\mathcal{D}$, then $\operatorname{Lat}(\mathcal{A})$ denotes the set of all projections $e$ of $\mathcal{D}$ such that $(1-e) a e=0$ for every $a \in \mathcal{A}$, and let $\mathcal{A l g}(\operatorname{Lat}(\mathcal{A}))$ be the subalgebra of $\mathcal{D}$ equal to

$$
\mathcal{A l g}(\operatorname{Lat}(\mathcal{A}))=\{y \in \mathcal{D}:(1-e) y e=0, \forall e \in \operatorname{Lat}(\mathcal{A})\} .
$$

Note that, as remarked in [1] page $344, \operatorname{Lat}(\mathcal{A})$ need not form a lattice, but we will follow the notation of previous authors.

Notice that if $\operatorname{Proj}(\mathcal{D})$ is the set of projections of $\mathcal{D}$ and if $\mathcal{A}$ is a sub- $\mathrm{C}^{*}$-algebra of $\mathcal{D}$, then $\operatorname{Lat}(\mathcal{A})=\left\{e \in \operatorname{Proj}(\mathcal{D}): e \in \mathcal{A}^{\prime}\right\}$ and $\left(\mathcal{A}^{\prime} \cap \mathcal{D}\right)^{\prime} \cap \mathcal{D} \subseteq \mathcal{A l g}(\operatorname{Lat}(\mathcal{A}))$.

In the proof of the last theorem (Theorem 4.3), in order to emphasize the ambient algebra $\mathcal{D}$, we will write instead $\operatorname{Lat}_{\mathcal{D}}(\mathcal{A})$ and $\mathcal{A} l g_{\mathcal{D}}\left(\operatorname{Lat}_{\mathcal{D}}(\mathcal{A})\right)$. For the rest of the paper, since the ambient algebra is clear, we will drop the subscript " $\mathcal{D}$ ".

Lemma 3.1. Let $\mathcal{D}$ be a unital $C^{*}$-algebra and $\mathcal{A}$ be a subalgebra of $\mathcal{D}$. Then for any $d \in \mathcal{D}$,

$$
\sup _{e \in \operatorname{Lat}(\mathcal{A})}\|(1-e) \operatorname{de}\| \leq \operatorname{dist}(d, \mathcal{A}) .
$$

Proof. As for any $a \in \mathcal{A}$ and $e \in \operatorname{Lat}(\mathcal{A})$,

$$
\|(1-e) d e\|=\|(1-e)(d-a) e\| \leq\|d-a\|,
$$

the lemma is verified. 
The proof of the next lemma is contained in the first part of the proof of [1, Corollary 2, pages 344 and 345 .

Lemma 3.2. Let $\mathcal{C}$ be a unital separable $C^{*}$-algebra and $\mathcal{A}$ be a unital subalgebra of $\mathcal{C}$.

Then for any $c \in \mathcal{C}$, there exist a unital ${ }^{*}$-representation $\psi: \mathcal{C} \rightarrow \mathbb{B}\left(\mathcal{H}_{\psi}\right)$ on a separable infinite dimensional Hilbert space $\mathcal{H}_{\psi}$ and a projection $p \in \mathbb{B}\left(\mathcal{H}_{\psi}\right)$ such that

(1) $p \in \operatorname{Lat}(\psi(\mathcal{A}))$, i.e., $(1-p) \psi(a) p=0$ for all $a \in \mathcal{A}$, and

(2) $\|(1-p) \psi(c) p\|=\|(1-p) \psi(c) p\|_{\text {ess }} \geq \operatorname{dist}(c, \mathcal{A})$, where the $\|\cdot\|_{\text {ess }}$ denotes the essential norm.

Lemma 3.3. Let $\mathcal{B}$ be a separable stable $C^{*}$-algebra, and let $\mathcal{A}$ be a separable norm-closed unital subalgebra of the corona algebra $\mathcal{M}(\mathcal{B}) / \mathcal{B}$.

If $x \in \mathcal{M}(\mathcal{B}) / \mathcal{B}$ is an element such that the inclusion of the $C^{*}$-algebra $C^{*}(x, \mathcal{A})$ generated by $x$ and $\mathcal{A}$ in $\mathcal{M}(\mathcal{B}) / \mathcal{B}$ is purely large, then there exists a projection $p \in \mathcal{M}(\mathcal{B}) / \mathcal{B}$ such that

(1) $p \in \operatorname{Lat}(\mathcal{A})$, and

(2) $\|(1-p) x p\|=\operatorname{dist}(x, \mathcal{A})$.

Proof. By Lemma 3.1, it suffices to find a projection $p \in \operatorname{Lat}(\mathcal{A})$ such that

$$
\|(1-p) x p\| \geq \operatorname{dist}(x, \mathcal{A}) .
$$

Since $\mathcal{B}$ is stable, we may replace $\mathcal{B}$ by $\mathcal{B} \otimes \mathcal{K}$, where $\mathcal{K}$ is the $\mathrm{C}^{*}$-algebra of compact operators on a separable infinite dimensional Hilbert space $\mathcal{H}$.

Let us denote by $\mathcal{C}$ the separable unital sub-C*-algebra of $\mathcal{M}(\mathcal{B} \otimes \mathcal{K}) /(\mathcal{B} \otimes \mathcal{K})$ generated by $x$ and $\mathcal{A}$.

Since $\mathcal{M}(\mathcal{K})=\mathcal{H}$, let us denote by $\sigma: \mathcal{C} \rightarrow \mathcal{M}(\mathcal{K})$ the unital *-homomorphism given by Lemma 3.2. and by $q_{0} \in \mathcal{M}(\mathcal{K})$ the projection such that

i. $\left(1-q_{0}\right) \sigma(a) q_{0}=0$, for all $a \in \mathcal{A}$, and

ii. $\left\|\left(1-q_{0}\right) \sigma(x) q_{0}\right\|=\left\|\left(1-q_{0}\right) \sigma(x) q_{0}+\mathcal{K}\right\|_{\mathcal{M}(\mathcal{K}) / \mathcal{K}} \geq \operatorname{dist}(x, \mathcal{A})$.

Note that since $\mathcal{K}$ is nuclear, the unital trivial extension

$$
\mathcal{C} \rightarrow \mathcal{M}(\mathcal{B} \otimes \mathcal{K}) / \mathcal{B} \otimes \mathcal{K}: c \mapsto \pi\left(1_{\mathcal{M}(\mathcal{B})} \otimes \sigma(c)\right)
$$

is weakly nuclear, where $\pi$ denotes the canonical surjection from $\mathcal{M}(\mathcal{B} \otimes \mathcal{K})$ onto the corona algebra $\mathcal{M}(\mathcal{B} \otimes \mathcal{K}) / \mathcal{B} \otimes \mathcal{K}$.

For any two isometries $S, T \in \mathcal{M}(\mathcal{B} \otimes \mathcal{K})$ such that $1_{\mathcal{M}(\mathcal{B} \otimes \mathcal{K})}=S S^{*}+T T^{*}$, let $s=\pi(S)$ and $t=\pi(T)$ denote the corresponding two isometries of $\mathcal{M}(\mathcal{B} \otimes \mathcal{K}) / \mathcal{B} \otimes \mathcal{K}$.

As by assumption the inclusion of $\mathcal{C}$ in $\mathcal{M}(\mathcal{B} \otimes \mathcal{K}) / \mathcal{B} \otimes \mathcal{K}$ is purely large, and as the trivial extension (11) is weakly nuclear, then by Theorem 2.2. there exists a unitary $U \in \mathcal{M}(\mathcal{B} \otimes \mathcal{K})$ such that for all $c \in \mathcal{C}$,

$$
c=u\left(s c s^{*}+t \pi\left(1_{\mathcal{M}(\mathcal{B})} \otimes \sigma(c)\right) t^{*}\right) u^{*}
$$

where $u=\pi(U)$.

Let $p$ be the projection of $\mathcal{M}(\mathcal{B} \otimes \mathcal{K}) /(\mathcal{B} \otimes \mathcal{K})$ given by

$$
p={ }_{d f} u t \pi\left(1_{\mathcal{M}(\mathcal{B})} \otimes q_{0}\right) t^{*} u^{*},
$$

and let us show that $p$ is the required projection.

As $(1-p) a p=u t \pi\left(1_{\mathcal{M}(\mathcal{B})} \otimes\left(1_{\mathcal{M}(\mathcal{K})}-q_{0}\right) \sigma(a) q_{0}\right) t^{*} u^{*}=0$ for all $a \in \mathcal{A}$, we have that $p \in \operatorname{Lat}(\mathcal{A})$. 
Moreover,

$$
\begin{aligned}
& \|(1-p) x p\| \\
= & \left\|(1-p) u t \pi\left(1_{\mathcal{M}(\mathcal{B})} \otimes \sigma(x)\right) t^{*} u^{*} u t \pi\left(1_{\mathcal{M}(\mathcal{B})} \otimes q_{0}\right) t^{*} u^{*}\right\| \\
= & \left\|(1-p) u t \pi\left(1_{\mathcal{M}(\mathcal{B})} \otimes \sigma(x) q_{0}\right)\right\| \\
= & \left\|\left[u s s^{*} u^{*}+u t \pi\left(1_{\mathcal{M}(\mathcal{B})} \otimes\left(1_{\mathcal{M}(\mathcal{K})}-q_{0}\right)\right) t^{*} u^{*}\right] u t \pi\left(1_{\mathcal{M}(\mathcal{B})} \otimes \sigma(x) q_{0}\right)\right\| \\
= & \left\|u t \pi\left(1_{\mathcal{M}(\mathcal{B})} \otimes\left(1_{\mathcal{M}(\mathcal{K})}-q_{0}\right) \sigma(x) q_{0}\right)\right\| \\
= & \left\|\left(1-q_{0}\right) \sigma(x) q_{0}+\mathcal{K}\right\|_{\mathcal{M}(\mathcal{K}) / \mathcal{K}} \\
\geq & \operatorname{dist}(x, \mathcal{A}) \text { by ii). }
\end{aligned}
$$

Theorem 3.4. Suppose that either $\mathcal{B} \cong \mathcal{K}$ or $\mathcal{B}$ is a separable simple stable purely infinite $C^{*}$-algebra. Let $\mathcal{A}$ be a norm-closed separable unital subalgebra of the corona algebra $\mathcal{M}(\mathcal{B}) / \mathcal{B}$, and $\operatorname{Lat}(\mathcal{A})$ be the set of all projections e $\in \mathcal{M}(\mathcal{B}) / \mathcal{B}$ such that $(1-e) a e=0$, for all $a \in \mathcal{A}$.

Then the algebra of all elements of $\mathcal{M}(\mathcal{B}) / \mathcal{B}$ leaving invariant each projection of $\boldsymbol{L a t}(\mathcal{A})$ is equal to $\mathcal{A}$.

As for any sub-C*-algebra $\mathcal{A}$ of a unital $\mathrm{C}^{*}$-algebra $\mathcal{D}$, we have

$$
\mathcal{A} \subseteq\left(\mathcal{A}^{\prime} \cap \mathcal{M}(\mathcal{B}) / \mathcal{B}\right)^{\prime} \cap \mathcal{M}(\mathcal{B}) / \mathcal{B} \subseteq \mathcal{A l g}(\operatorname{Lat}(\mathcal{A})),
$$

the following corollary is a direct consequence of Theorem 3.4

Corollary 3.5. If $\mathcal{B} \cong \mathcal{K}$ or $\mathcal{B}$ is a separable simple stable purely infinite $C^{*}$ algebra, and $\mathcal{A}$ is a separable unital sub- $C^{*}$-algebra of $\mathcal{M}(\mathcal{B}) / \mathcal{B}$, then $\mathcal{A}$ is equal to its relative bicommutant, i.e.,

$$
\mathcal{A}=\left(\mathcal{A}^{\prime} \cap \mathcal{M}(\mathcal{B}) / \mathcal{B}\right)^{\prime} \cap \mathcal{M}(\mathcal{B}) / \mathcal{B} .
$$

Proof of Theorem 3.4. Let

$$
\mathcal{A} l g(\operatorname{Lat}(\mathcal{A}))=_{d f}\{x \in \mathcal{M}(\mathcal{B}) / \mathcal{B}:(1-e) x e=0, \forall e \in \operatorname{Lat}(\mathcal{A})\}
$$

denote the algebra of elements of $\mathcal{M}(\mathcal{B}) / \mathcal{B}$ leaving invariant Lat $(\mathcal{A})$.

As $\mathcal{A}$ is clearly contained in $\mathcal{A l g}(\operatorname{Lat}(\mathcal{A}))$, let us prove the other inclusion.

Let $x \in \mathcal{A l g}(\operatorname{Lat}(\mathcal{A}))$ and let $\mathcal{C}=C^{*}(x, \mathcal{A})$ be the separable unital sub-C. algebra of $\mathcal{M}(\mathcal{B}) / \mathcal{B}$ generated by $x$ and $\mathcal{A}$. By Proposition 2.4 the inclusion of $\mathcal{C}$ into $\mathcal{M}(\mathcal{B}) / \mathcal{B}$ is purely large. Hence, by Lemma 3.3, there exists a projection $p \in \operatorname{Lat}(\mathcal{A}) \subset \mathcal{M}(\mathcal{B}) / \mathcal{B}$ such that

$$
\|(1-p) x p\|=\operatorname{dist}(x, \mathcal{A}) .
$$

As $x \in \mathcal{A l g}(\operatorname{Lat}(\mathcal{A}))$ and $\mathcal{A}$ is closed, $x \in \mathcal{A}$.

\section{Pedersen's Question}

Theorem 4.3, the main result of this section generalizes Theorem 3.4, by relaxing the separability condition on the $\mathrm{C}^{*}$-algebra $\mathcal{B}$. Then in Corollary 4.4, we give a complete answer in the stable case to Pedersen's question in [17.

Before stating and proving these results, let us first introduce some preliminaries needed below. 
Lemma 4.1. Let $\mathcal{B}$ be a simple, purely infinite $C^{*}$-algebra, and let $a, b \in \mathcal{B}_{+}$, $\|a\|=\|b\|=1$.

Then for any $0<\delta<1$, and for any $\epsilon>0$, there exists $x \in \mathcal{B}$ such that

$$
\left\|a-x b x^{*}\right\| \leq \epsilon \text { and }\|x\|^{2} \leq \frac{1}{\delta}
$$

Proof. For $0<\delta<1$, fixed, we have $\left\|b-(b-\delta)_{+}\right\| \leq \delta$ and as $\mathcal{B}$ is simple purely infinite, $a \preceq(b-\delta)_{+}$. Then for any $\epsilon>0$, there exists by [11], Lemma 2.2, $x \in \mathcal{B}$ with $\|x\|^{2} \leq \frac{1}{\delta}$ and $(a-\epsilon)_{+}=x b x^{*}$. Then $\left\|a-x b x^{*}\right\| \leq\left\|a-(a-\epsilon)_{+}\right\| \leq \epsilon$.

Let $\mathcal{C}$ be a separable sub-C*-algebra of a $\sigma$-unital, stable, simple, purely infinite $\mathrm{C}^{*}$-algebra $\mathcal{B}$, and let $\left\{c_{n}\right\}_{n \geq 1}$ be a countable dense subset of the unit sphere of $\mathcal{C}_{+}$.

By Lemma 4.1, for each $n, m$ and $k \geq 1$, there exists $y_{n, m, k} \in \mathcal{B}$, such that $\left\|y_{n, m, k}\right\|^{2} \leq \frac{3}{2}$ and

$$
\left\|y_{n, m, k} c_{n} y_{n, m, k}^{*}-c_{m}\right\|<\frac{1}{k}
$$

Then the separable sub- $\mathrm{C}^{*}$-algebra $\widetilde{\mathcal{C}}$ of $\mathcal{B}$ generated by $\mathcal{C}$ and $\left\{y_{n, m, k}: n, m, k \geq\right.$ $1\}$ is said to be obtained from $\mathcal{C}$ by a PI-operation.

Using this construction inductively, we have the following:

Proposition 4.2. Let $\mathcal{B}$ be a $\sigma$-unital, stable, simple purely infinite $C^{*}$-algebra, and let $\mathcal{C} \subseteq \mathcal{B}$ be a separable sub- $C^{*}$-algebra.

Then there exists a separable, stable, simple, purely infinite sub-C*-algebra $\mathcal{D}$ of $\mathcal{B}$ containing $\mathcal{C}$.

Proof. Let $b \in \mathcal{B}_{+}$be a strictly positive element, and let $\mathcal{C}_{0}$ be the (separable) sub$\mathrm{C}^{*}$-algebra generated by $\mathcal{C}$ and $b$. By [19] Corollary 2.3, any sub-C*-algebra of $\mathcal{B}$ containing $\mathcal{C}_{0}$ is stable. Then construct an increasing sequence $\left\{\mathcal{C}_{l}\right\}_{l \geq 0}$ of separable sub- $\mathrm{C}^{*}$-algebras of $\mathcal{B}$ such that $\mathcal{C}_{l+1}$ is obtained from $\mathcal{C}_{l}$ by a PI-operation, and $\mathcal{D}={ }_{d f} \overline{\bigcup_{l>1} \mathcal{C}_{l}}$. Then $\mathcal{D}$ contains $\mathcal{C}_{0}$, is stable, and simple (by similar argument as in [3] II.8.5.6).

To prove that $\mathcal{D}$ is purely infinite, it is enough to show that for any $c, d \in \mathcal{D}_{+}$, $\|c\|=\|d\|=1$, for any $\epsilon>0$, there exists $y \in \mathcal{D}$ such that

$$
\left\|y c y^{*}-d\right\|<\epsilon .
$$

For $l \geq 0$, let $\left\{c_{n}^{l}\right\}_{n \geq 1}$ denote the dense sequence of the unit sphere of $\left(\mathcal{C}_{l}\right)_{+}$, used in the construction of $\mathcal{C}_{l+1}$. Then choose $l \geq 1$ and $c_{n}^{l}, c_{m}^{l} \in\left(\mathcal{C}_{l}\right)_{+}$such that

$$
\left\|c-c_{n}^{l}\right\| \leq \frac{\epsilon}{4} \text { and }\left\|d-c_{m}^{l}\right\| \leq \frac{\epsilon}{3} .
$$

By definition of $\mathcal{C}_{l+1}$, there exists a $y \in \mathcal{C}_{l+1},\|y\|^{2} \leq \frac{3}{2}$ such that

$$
\left\|c_{m}^{l}-y c_{n}^{l} y^{*}\right\| \leq \frac{\epsilon}{3} \text {. }
$$

Hence,

$$
\left\|d-y c y^{*}\right\| \leq \frac{\epsilon}{3}+\frac{\epsilon}{3}+\left\|y\left(c_{n}^{l}-c\right) y^{*}\right\| \leq \frac{\epsilon}{3}+\frac{\epsilon}{3}+\frac{3}{2} * \frac{\epsilon}{4} \leq \epsilon .
$$


Theorem 4.3. Let $\mathcal{B}$ be either the compact operators $\mathcal{K}$ or a $\sigma$-unital, stable, simple, purely infinite $C^{*}$-algebra.

Let $\mathcal{A}$ be a separable, norm-closed, unital subalgebra of $\mathcal{M}(\mathcal{B}) / \mathcal{B}$.

Then

$$
\mathcal{A}=\mathcal{A l g}(\boldsymbol{L a t}(\mathcal{A}))
$$

Recall (14. Theorem 3.8) that if $\mathcal{B}$ is a stable $\sigma$-unital $\mathrm{C}^{*}$-algebra, then the corona algebra $\mathcal{M}(\mathcal{B}) / \mathcal{B}$ is simple if and only if either $\mathcal{B} \cong \mathcal{K}$ or $\mathcal{B}$ is simple purely infinite.

Then the following corollary, which follows immediately from Theorem 4.3, resolves the stable case of Pedersen's question.

Corollary 4.4. Suppose that either $\mathcal{B} \cong \mathcal{K}$ or $\mathcal{B}$ is a $\sigma$-unital stable simple purely infinite $C^{*}$-algebra.

Let $\mathcal{A}$ be a separable unital sub-C $C^{*}$-algebra of $\mathcal{M}(\mathcal{B}) / \mathcal{B}$.

Then

$$
\left(\mathcal{A}^{\prime} \cap \mathcal{M}(\mathcal{B}) / \mathcal{B}\right)^{\prime} \cap \mathcal{M}(\mathcal{B}) / \mathcal{B}=\mathcal{A}
$$

In the proof of Theorem 4.3, we will need the following notions and results from 11:

Let $\mathcal{B}$ be a $\sigma$-unital simple $\mathrm{C}^{*}$-algebra. Then a sequence $\left\{x_{l}\right\}_{l \geq 1}$ of $\mathcal{B}_{+}$gives a bidiagonal series $X$ if $X=\sum_{l \geq 1} x_{l}$ converges in the strict topology and $x_{k} x_{l}=0$ for all $|k-l| \geq 2$.

If $T \in \mathcal{M}(\mathcal{B})_{+}$, then by [11], Theorem 4.2, for any $\epsilon>0$, there exist a bidiagonal series $\sum_{k \geq 1} t_{k}$ and a self-adjoint element $a_{\epsilon} \in \mathcal{B}$ with $\left\|a_{\epsilon}\right\|<\epsilon$ and

$$
T=\sum_{k \geq 1} t_{k}+a_{\epsilon}
$$

Proof of Theorem 4.3. To simplify notation, let us denote $\mathcal{C}(\mathcal{B})={ }_{d f} \mathcal{M}(\mathcal{B}) / \mathcal{B}$.

As $\mathcal{A}$ is already contained in $\mathcal{A l g}_{\mathcal{C}(\mathcal{B})}\left(\operatorname{Lat}_{\mathcal{C}(\mathcal{B})}(\mathcal{A})\right)$, let us prove that if $x \in$ $\mathcal{A} \lg _{\mathcal{C}(\mathcal{B})}\left(\operatorname{Lat}_{\mathcal{C}(\mathcal{B})}(A)\right)$ then $x \in \mathcal{A}$.

Let $\left\{a_{l}\right\}_{l=1}^{\infty}$ be a dense sequence in $\mathcal{A}$ and choose $X$ and $A_{l}$ in $\mathcal{M}(\mathcal{B})$ such that $\pi(X)=x$ and $\pi\left(A_{l}\right)=a_{l}$ for $l \geq 1$ (where $\pi: \mathcal{M}(\mathcal{B}) \rightarrow \mathcal{M}(\mathcal{B}) / \mathcal{B}$ is the canonical quotient map). By [11, Theorem 4.2, there exist for $l \geq 1$ and $1 \leq j \leq 4$, bidiagonal series $X_{j}$ and $A_{l, j}$ in $\mathcal{M}(\mathcal{B})_{+}$and $y, c_{l} \in \mathcal{B}$, such that

$$
X=\sum_{j=1}^{4} i^{j} X_{j}+y \text { and } A_{l}=\sum_{j=1}^{4} i^{j} A_{l, j}+c_{l},
$$

Then, let us write $X_{j}=\sum_{n \geq 1} x_{j, n}$ and $A_{l, j}=\sum_{n \geq 1} d_{l, j, n}$, where for $1 \leq j \leq 4$ and $l \geq 1,\left\{x_{j, n}, d_{l, j, n}: n \geq 1\right\} \subset \mathcal{B}_{+}$and the series converge in the strict topology.

Let $b \in \mathcal{B}_{+}$be strictly positive. Let $\mathcal{C}$ denote the separable sub- $\mathrm{C}^{*}$-algebra of $\mathcal{B}$, generated by $\left\{b, y, c_{l}, x_{j, n}, d_{l, j, n}: 1 \leq j \leq 4, l, n \geq 1,\right\}$. By Proposition 4.2, let $\mathcal{D}$ be a separable, stable, simple, purely infinite sub- $\mathrm{C}^{*}$-algebra of $\mathcal{B}$ containing $\mathcal{C}$.

For $l \geq 1$ and $1 \leq j \leq 4$, by construction, $X_{j}$ and $A_{l, j}$ belong to $\mathcal{M}(\mathcal{D})$, and therefore, $X, A_{l} \in \mathcal{M}(\mathcal{D})$. 
Since $b \in \mathcal{D}$, the inclusion $\mathcal{D} \subseteq \mathcal{B}$ induces the (unital) inclusion $\mathcal{M}(\mathcal{D}) \subseteq \mathcal{M}(\mathcal{B})$ and $\mathcal{M}(\mathcal{D}) / \mathcal{D} \subseteq \mathcal{M}(\mathcal{B}) / \mathcal{B}$. Moreover, $x=\pi(X)$ and $a_{l}=\pi\left(A_{l}\right)$, for $l \geq 1$, belong to $\mathcal{M}(\mathcal{D}) / \mathcal{D}$.

Since $x \in \mathcal{A l g}_{\mathcal{C}(\mathcal{B})}\left(\operatorname{Lat}_{\mathcal{C}(\mathcal{B})}(\mathcal{A})\right) \cap \mathcal{C}(\mathcal{D}), x \in \mathcal{A l g} g_{\mathcal{C}(\mathcal{D})}\left(\operatorname{Lat}_{\mathcal{C}(\mathcal{D})}(\mathcal{A})\right)$ and by Theo$\operatorname{rem} 3.4 x \in \mathcal{A}$.

Farah asked whether $\left(A^{\prime} \cap \mathcal{D}^{* *}\right)^{\prime} \cap \mathcal{D}=\mathcal{A}$ for every unital simple purely infinite separable $\mathrm{C}^{*}$-algebra $\mathcal{D}$ and $\mathcal{A}$ any unital sub- $\mathrm{C}^{*}$-algebra of $\mathcal{D}$ ([7, Question 5.2). We note that, by arguments similar to those for Proposition 4.2 and Theorem 4.3. this question is equivalent to the same question but without the requirement that $\mathcal{D}$ be separable. We have actually answer Farah's question in the affirmative for the case where $\mathcal{D}$ is a nonseparable simple purely infinite $\mathrm{C}^{*}$-algebra of the form $\mathcal{M}(\mathcal{B}) / \mathcal{B}$ where $\mathcal{B}$ is $\sigma$-unital and stable. Here is the short argument: If $x \in$ $\left(\mathcal{A}^{\prime} \cap(\mathcal{M}(\mathcal{B}) / \mathcal{B})^{* *}\right)^{\prime} \cap \mathcal{M}(\mathcal{B}) / \mathcal{B}$, then by [7, Proposition $5.3, x \in\left(\mathcal{A}^{\prime} \cap(\mathcal{M}(\mathcal{B}) / \mathcal{B})\right)^{\prime} \cap$ $\mathcal{M}(\mathcal{B}) / \mathcal{B}$, and so by Corollary 4.4, $x \in \mathcal{A}$.

\section{REFERENCES}

[1] W. Arveson, Notes on extensions of $C^{*}$-algebras. Duke Math. J. 44 (1977), no. 2, 329-355.

[2] B. Blackadar, K-theory for operator algebras. Second edition. Mathematical Sciences Research Institute Publications, 5. Cambridge University Press, Cambridge, 1998.

[3] B. Blackadar, Operator algebras. Theory of $\mathrm{C}^{*}$-algebras and von Neumann algebras. Encyclopedia of Mathematical Sciences, 122. Operator algebras and noncommutative geometry, III. Springer-Verlag, Berlin, 2006.

[4] L. G. Brown, R. G. Douglas and P. A. Fillmore, Unitary equivalence modulo the compact operators and extensions of $C^{*}$-algebras. Proceedings of a Conference on Operator Theorem (Dalhouse Univ., Halifax, N.S., 1973), pp58-128. Lecture Notes in Math., Vol 345, Springer, Berlin, 1973.

[5] G. A. Elliott and D. Kucerovsky, An abstract Voiculescu-Brown-Douglas-Fillmore absorption theorem. Pacific J. Math. 198 (2001), no. 2, 385-409.

[6] G. A. Elliott and D. Kucerovsky, A relative double commutant theorem for hereditary sub-C*algebras. C. R. Math. Acad. Sci. Soc. R. Can. 29 (2007), no. 1, 22-27.

[7] I. Farah, A new bicommutant theorem. Accepted for publication at the Pacific Journal of Mathematics. https://arxiv.org/abs/1603.02105.

[8] D. Hadwin, An asymptotic double commutant theorem for $C^{*}$-algebras. Trans. Amer. Math. Soc. 244 (1978), 273-297.

[9] D. Hadwin, Approximate double commutants in von Neumann algebras and $C^{*}$-algebras. Oper. Matrices 8 (2014), no. 3, 623-633.

[10] D. Hadwin and J. Shen, Approximate double commutants and distance formulas. Oper. Matrices 8 (2014), no. 2, 529-553.

[11] V. Kaftal, P. W. Ng and S. Zhang, Strict comparison of positive elements in multiplier algebras. Canad. J. Math. 69 (2017), no. 2, 373-407.

[12] G. G. Kasparov, Hilbert $C^{*}-$ modules: theorems of Stinespring and Voiculescu. J. Operator Theory 4 (1980), no. 1, 133-150.

[13] D. Kucerovsky, Double commutants of hereditary subalgebras of a corona. J. Funct. Anal. 219 (2005), no. 2, 245-254.

[14] H. Lin, Simple $C^{*}$-algebras with continuous scales and simple corona algebras. Proceedings of the AMS. 112 (1991), no. 3, 871-880.

[15] H. Lin, Simple corona algebras. Proceedings of the American Mathematical Society 132 (2004), no. 11, 3215-3224.

[16] H. Lin, Stable approximate unitary equivalence of homomorphisms. J. Operator Theory 47 (2002), no. 2, 343-378.

[17] G. Pedersen, The corona construction. Operator Theory: Proceedings of the 1988 GPOTSWabash Conference (Indianapolis, IN, 1988), 49-92, Pitman Res. Notes Math. Ser., 225, Longman Sci. Tech., Harlow, 1990. 
[18] M. Rordam, Ideals in the multiplier algebra of a stable algebra. J. Operator Theory 25 (1991), $283-298$.

[19] M. Rordam, Stable $C^{*}$-algebras. Operator Algebras and Applications, 177-199, Adv. Stud. Pure Math., 38 Math. Soc. Japan, Tokyo, 2004.

[20] K. Jensen and K. Thomsen, Elements of KK-theory. Mathematics: Theory and Applications. Birkhaeuser Boston, Inc., Boston, MA, 1991.

[21] D. Voiculescu, A noncommutative Weyl-von Neumann theorem. Rev. Roumaine Math. Pures Appl. 21 (1976), 97-113.

[22] S. Zhang, On the structure of projections and ideals of corona algebras. Canad. J. Math. 41 (1989) 721-742.

[23] S. Zhang, A Riesz decomposition property and ideal structure of multiplier algebras. J. Operator Theory 24 (1990), 209-225.

Department of Mathematics and Statistics, University of Ottawa, 585 King Edward Ave., Ottawa, Ontario, K1N 6N5, Canada

E-mail address: giordano@uOttawa.ca

Department of Mathematics, University of Louisiana at Lafayette, 217 Maxim Doucet

Hall, P. O. Box 43568, Lafayette, Louisiana, 70504-3568, USA

E-mail address: png@louisiana.edu 